\title{
The 40th anniversary of the Ai Project: the commemorative gift is a silk scarf painted by $A i$ the chimpanzee
}

\author{
Tetsuro Matsuzawa ${ }^{1,2,3}$
}

Published online: 14 March 2017

(C) Japan Monkey Centre and Springer Japan 2017

An infant female chimpanzee named "Ai" came to Kyoto University Primate Research Institute (KUPRI) on 10 November 1977. She was estimated to be about 1 year of age when she first arrived, and this year, 2017, is the 40th anniversary of the "Ai Project."

At the age of 1.5 years old, on 15 April 1978, Ai pressed a key of a keyboard attached to computer-controlled apparatus for the very first time. Since then, she has participated in various kinds of behavioral cognitive experiments. The first marked achievement in behavioral cognitive studies on chimpanzees was the seminal paper published in Nature in 1985 reporting their use of numbers (Matsuzawa 1985). The cognitive studies carried out with the chimpanzees housed at KUPRI are summarized in Matsuzawa (2003) and Matsuzawa et al. (2006) (Fig. 1).

The Ai Project is characterized by fully automated, computer-controlled experiments. This design allows the comparison of cognitive performance in humans and chimpanzees in the same place, using the same apparatus, following the same procedure. The experiments follow the approach of using psychophysical measurement to analyze perception, cognition, memory and other psychological functions. The project is aimed at showing the perception of the world by chimpanzees and comparing this to that of humans. The Ai Project led to the establishment of the discipline termed "comparative cognitive science" (CCS).

Tetsuro Matsuzawa

matsuzawa.tetsuro.8w@kyoto-u.ac.jp

Institute for Advanced Study, Kyoto University, Kyoto, Japan

2 Primate Research Institute, Kyoto University, Inuyama, Japan

3 Japan Monkey Centre, Inuyama, Japan
CCS initially involved highly objective, cognitive experiments in the laboratory. However, CCS is currently based on a parallel endeavor, pairing laboratory with field studies. These two different lines of research are complimentary. Fieldwork provides us with the basis to ask the right questions, i.e., those with ecological validity. For example, in Africa, we can learn about the lives of wild chimpanzees in their natural habitats, and the mind of the chimpanzee must be explored within the proper ecological and social contexts. Conversely, laboratory work can show us how to refine each distinct, field-observed episode into an organized understanding, even in the uncontrolled environment of wild habitats. For instance, through the introduction of field experiments, we established an outdoor laboratory.

\section{Participant observation}

I have carried out my fieldwork in Bossou and Nimba, Guinea, West Africa, since 1986 (Matsuzawa et al. 2011; Matsuzawa 2017). Fieldwork showed me how important social life is to chimpanzees, especially the mother-infant bond. Ai gave birth to her son, Ayumu, on 24 April 2000. At this point, we introduced a new research method called "participant observation," whereby researchers participated in the chimpanzees' daily lives in order to understand how the chimpanzee mind works in these every-day circumstances.

Scholars in the West had previously tried to compare humans and chimpanzees in a method called "cross-fostering", whereby a baby chimpanzee is raised in one's own home, with the aim of directly comparing infants of the two species, chimpanzee and human, by keeping them in the same rearing environment. Supposing that the human 


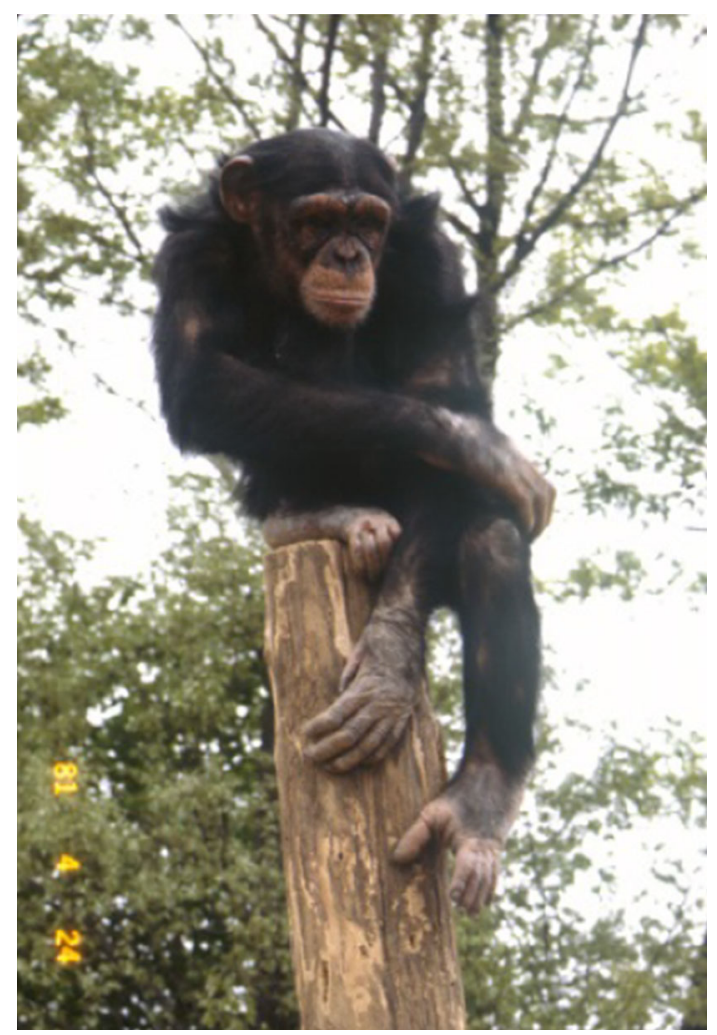

Fig. 1 The chimpanzee Ai at 4.5 years of age (photograph taken on 14 April 1981 by Tetsuro Matsuzawa)

infant starts speaking at around 1 year old, but that the chimpanzee infant does not, one may conclude that the capacity for speech is innate in humans, but not in chimpanzees. This illustrates the logic of this method. Such home-rearing experiments, with infant chimpanzees isolated from conspecifics, were carried out in the West during the 20th century, over a period of almost 100 years. However, it can be argued that this kind of comparison is unfair because in cross-fostering the human infant still has both her/his own parents, whereas the infant chimpanzee has no conspecific parent whatsoever. The infant chimpanzee is isolated from her/his biological mother as well as from other chimpanzees. It is not fair to isolate the infant chimpanzee in this way, to force her/him to adapt to life in a human environment, which entails adaptation to the environment of a different species, not that of its own species. Thus, cross-fostering is now recognized as unfair, unscientific, and unethical. One should not separate the infant chimpanzee from her/his mother. The infant needs to be raised by the mother chimpanzee to learn the essentials of survival from her. That is the lesson that I learned from my fieldwork observations, watching the social relationship between chimpanzee mother and infant.

The "participant observation" method is very different from the Western-style comparison, as the infant is not separated from her/his mother, who raises her own infant

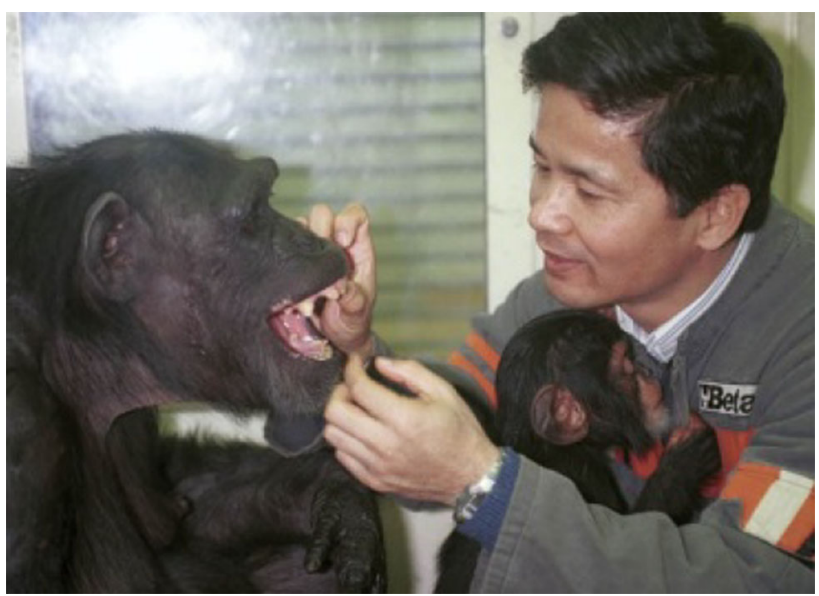

Fig. 2 The triadic relationship of researcher, mother chimpanzee Ai and infant chimpanzee Ayumu (photograph taken by Akihiro Hirata)

(Matsuzawa 2016). Participant observation consists of the triadic relationship between researcher, mother chimpanzee and infant chimpanzee (Fig. 2). Thanks to the long-standing relationship established between researcher and mother chimpanzee, the researcher can "request" that the mother chimpanzee helps him/her to study the chimpanzee infant.

In participant observation, the researcher endeavors to stay with the chimpanzees for as long as possible in the same living space, on a daily basis. For this purpose, we built a unique space called a "twin booth", in which two almost identical booths are placed side by side. There is a door between the two booths that can alternately be opened to connect the two areas, closed to separate the two individuals, or positioned half-open to allow the young chimpanzee to crawl from one area to the other while preventing the mother chimpanzee from entering the neighboring booth. This set-up results in intense interactions between the researcher and the two chimpanzees. However, the chimpanzees remain in the company of other chimpanzees for the majority of the daytime.

Participant observation using a twin booth also provides a unique possibility as a visiting researcher can occupy one booth and the mother-infant pair the other. Jane Goodall, as one of our visitors, enjoyed interacting with Ayumu, Ai's son (Fig. 3). Ayumu stayed with his mother in one booth at the beginning, then Ayumu, by his own choice, spontaneously crawled into the neighboring booth through the half-open door. Mother Ai, sitting in the neighboring booth, watched the scene through the separating transparent acrylic panel.

Regarding daily life, in comparison to the computercontrolled cognitive experiments, we have carried out fewer formal experiments by participant observation in a face-to-face context. The typical activity within the booth is drawing/painting, as $\mathrm{Ai}$ and other chimpanzees love to 


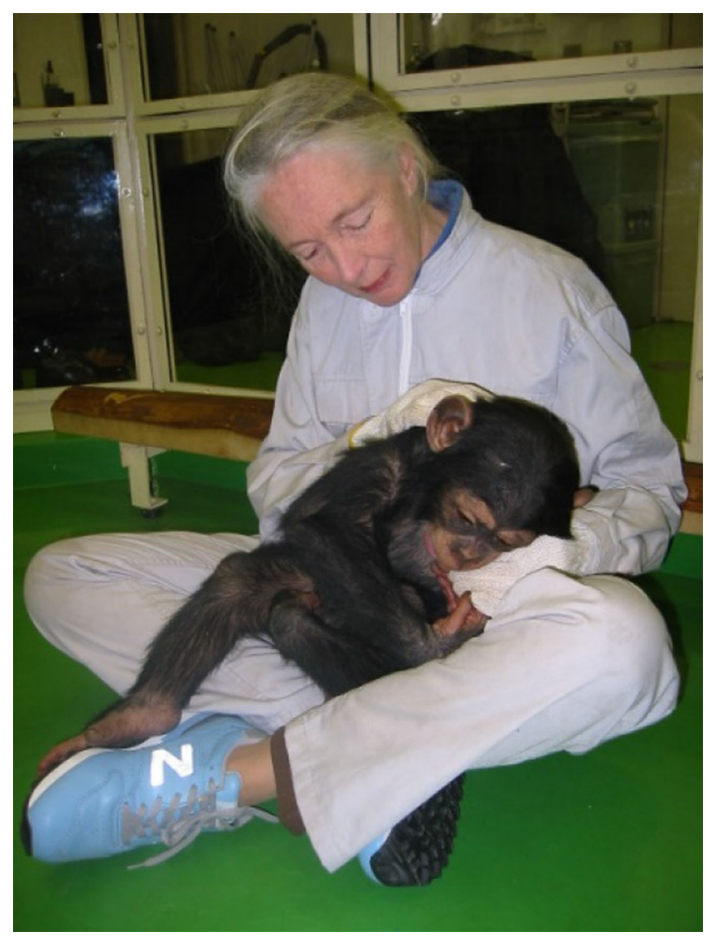

Fig. 3 Jane Goodall and Ayumu (photograph taken by Tetsuro Matsuzawa)

draw/paint, without any food reward (Matsuzawa 1996; Saito et al. 2014; Tanaka et al. 2003).

\section{Art meets ape}

$\mathrm{Ai}$ and other chimpanzees at KUPRI love to free-draw/paint. When some marker pens and a sheet of white paper are provided, without any instruction or molding, the chimpanzees love to push the marker pens against the blank paper, resulting in the outline of a picture. The feedback of the marker pen traces is sufficiently rewarding to maintain this drawing behavior in chimpanzees. This is a universal behavior among chimpanzees, and no food reward is necessary. The act of drawing is supported by intrinsic motivation, and there is a lot of evidence suggesting that great apes in general love to draw (Morris 1962).

Chimpanzee Ai drew from a very young age. I knew from personal experience that other great apes love to draw and paint too, so I had the idea of collecting the artwork of great apes. For this purpose, I asked Ai to draw and paint and used her creations as gifts to exchange for drawings made by ape friends. For example, I asked Roger and Deborah Fouts to send a drawing/painting made by Washoe the chimpanzee while I presented artwork created by Ai as a gift to them.

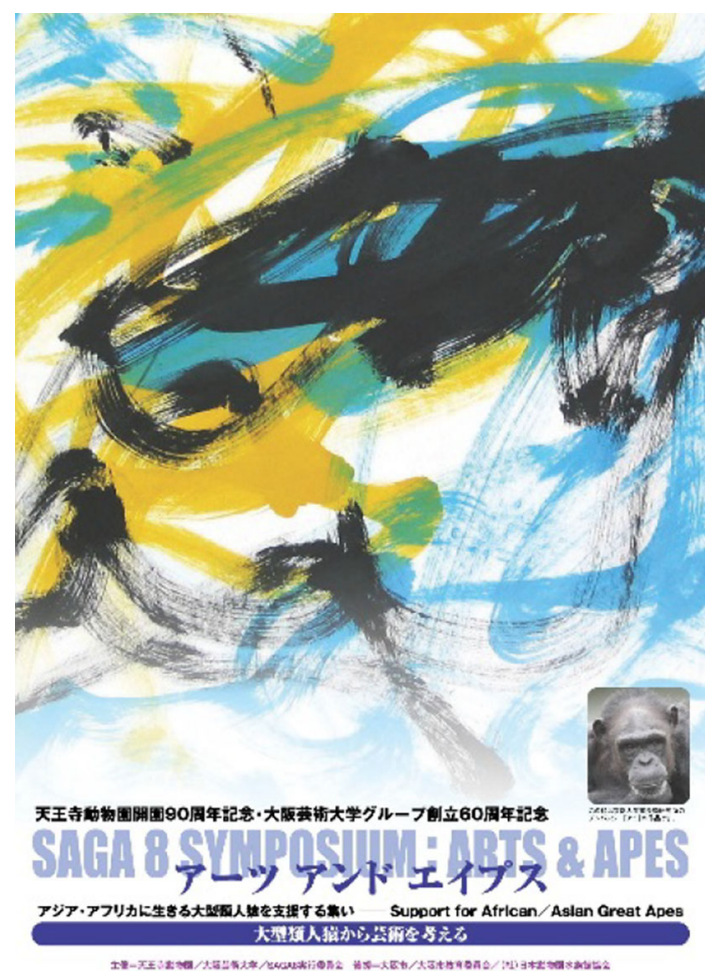

Fig. 4 The poster for the "Arts and Apes" exhibition held at the annual symposium of Support of African/Asian Great Apes (SAGA8) in 2005

Artwork made by Koko the Gorilla, from Francine Patterson, and from Kanzi the bonobo, through Sue Savage-Rumbaugh, were obtained in the same way. There was a very old female orangutan named Molly living at Ueno Zoo in Tokyo who loved to draw and paint too, so we also received her artwork in exchange for Ai's. In sum, we succeeded in building a unique collection of artwork created by great apes: chimpanzees, bonobos, gorillas, and orangutans.

Support of African/Asian Great Apes (SAGA) is an assemblage of people who care about great apes in the wild and in captivity (Matsuzawa 2016). SAGA was founded in 1998 and a meeting is held annually jointly at a university institute and zoo. This year, 2017, we will celebrate the 20th anniversary of SAGA. In 2005, we set up the first ever ape artwork exhibition, entitled "Arts and Apes" (Fig. 4), for the 8th Annual Saga symposium (SAGA 8), held in Osaka jointly by Osaka University of Arts and Tennoji Zoo.

Jane Goodall has been heavily involved since the founding of SAGA. She travels to Japan every year in November to attend every SAGA annual symposium. She took the opportunity to visit the Arts and Apes Exhibition during SAGA 8, really loved the idea of art by apes, and enjoying the variety and beauty of the great ape paintings (Fig. 5). 


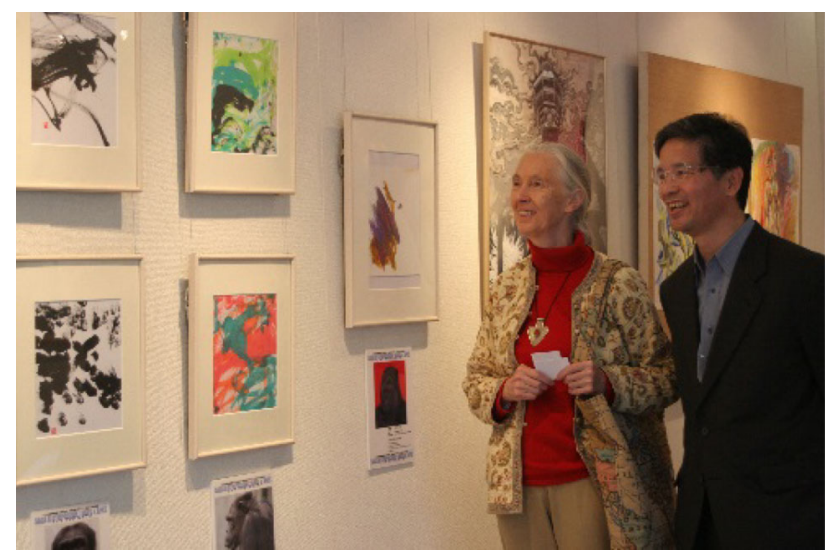

Fig. 5 Jane Goodall and the author enjoying the artwork of great apes (photograph provided by SAGA8)

\section{Free drawing by the KUPRI chimpanzees}

Chimpanzee Ai loves to draw and paint, and during daily interaction, we occasionally give her the opportunity to do this. Not only Ai but also other chimpanzees in the KUPRI community also seem to love drawing and painting. So far, I have tried out drawing/painting in a face-to-face context with nine chimpanzees, seven females and two males, Ai, Pendesa, Popo, Chloe, Cleo, Pan, Pal, Akira, and Ayumu.

My typical method is as follows; after the session of computer-controlled cognitive experiments, I enter the booth with the subject chimpanzee, taking art materials, marker pens, paint brushes, paint, sheets of paper, a water container, etc., with me. The marker pens are of 12 different colors.

In the standard free-drawing test, I place a sheet of white paper (B4; $257 \times 364 \mathrm{~mm})$ in front of the subject chimpanzee. Next, I hold the box of 12 marker pens and encourage the chimpanzee to take one of any color. The subject is free to choose any of the 12 colors and free to keep drawing as long as she/he wants. Sooner or later, the subject stops drawing by dropping the marker pen.

Next, I ask the chimpanzee, using spoken English and manual gestures, including pointing to the marker pen, whether she/he is sure that they wish to stop drawing. In many cases, the chimpanzee does not pick up the marker pen again, and drawing with that particular color is finished. In other cases, the chimpanzee picks up the marker pen once more and recommences drawing. When the chimpanzee stops drawing again, drawing in this color is finished and we move on to the next color choice. Thus, one can see an order of color preference based on free choice. The whole episode is video-recorded for future analysis of variables such as hand preference, grip type, and the sequential order of particular scribbles/traces, etc.

On special occasions, we ask the subject chimpanzee to use paint brushes to paint on paper of various different sizes. In this case, the procedure is very similar to that when using marker pens as drawing implements. When using paint brushes, the experimenter prepares the brushes, each loaded with paint of a particular color, in advance, and asks the subject to choose. There is also another variation of the painting session in which the experimenter, not the subject, chooses the color and sequence of use. In this case, the final painting is a product of the collaboration between the chimpanzee painter and the human designer.

As an example of one such special case, in April 2013, Prof. Kazuo Oike was appointed Dean of Kyoto University of Art and Design. Prof. Oike was the 24th president of Kyoto University and helped a great deal in the creation of the Wildlife Research Center (WRC) of the university, founded in 2008, which includes the Kumamoto Sanctuary, the first chimpanzee sanctuary in Japan. In this way, Prof. Oike contributed to promoting the welfare of captive chimpanzees. When Prof. Oike took up the position of dean, he requested a special gift from me: artwork by chimpanzee Ai. I asked Dr. Aya Saitoh, a specialist in the drawings of chimpanzees and children, to help me to set up a painting by $\mathrm{Ai}$.

In this session, Ai created a picture using paint and paint brushes on special paper called Shikishi $(242 \times 273 \mathrm{~mm})$. This particular painting was initially intended solely as a gift for Prof. Oike. The colors were not chosen by Ai, but were selected by Dr. Saitoh: Ai was given a paint brush with red paint and then one with black paint. It was
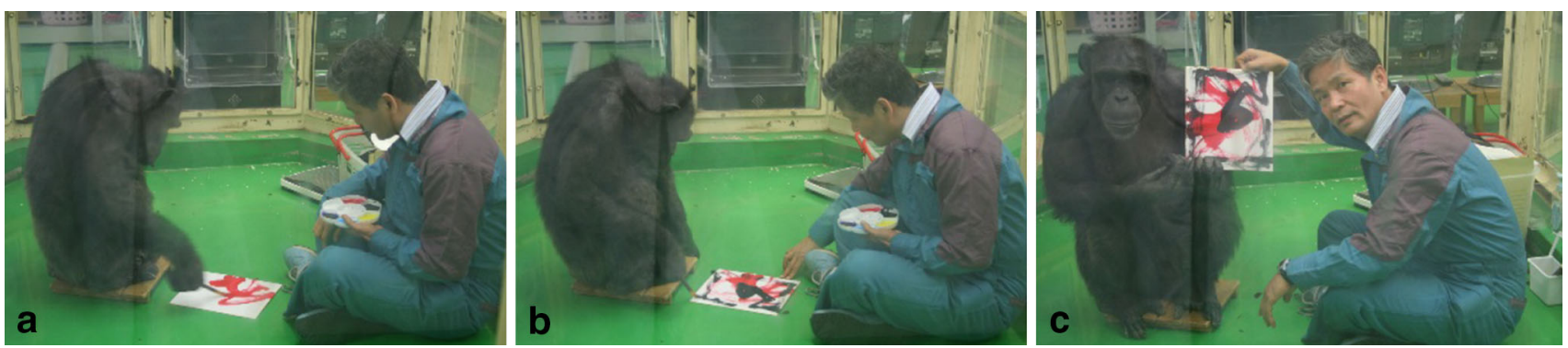

Fig. 6 Ai's painting process (photographs provided by the Primate Research Institute of Kyoto University) 


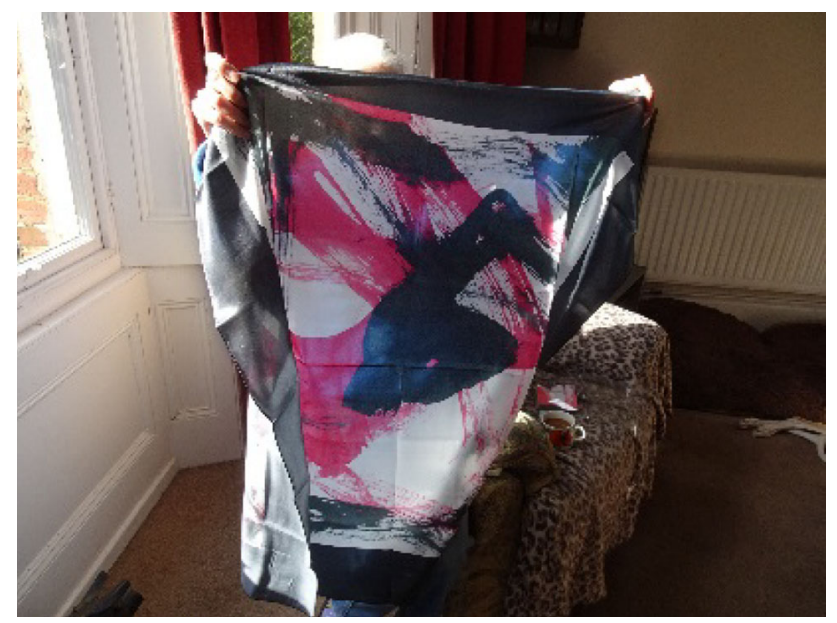

Fig. 7 Jane Goodall holds up the scarf printed with the design painted by chimpanzee Ai (photographs by Tetsuro Matsuzawa)

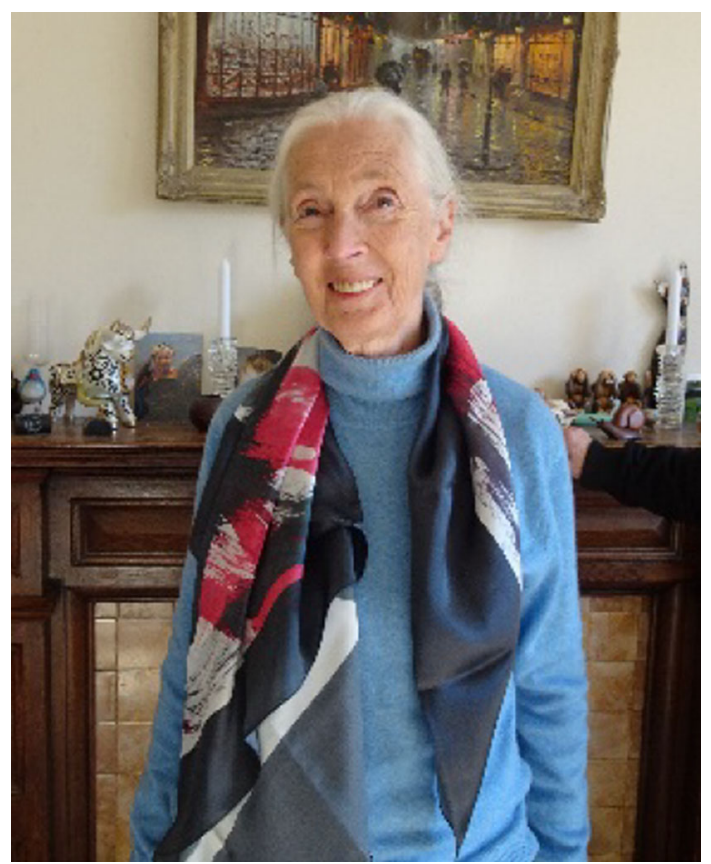

Fig. 8 Jane Goodall wearing the scarf (photographs by Tetsuro Matsuzawa)

chimpanzee Ai who made the decision of how exactly to apply the paint brushes to the paper (Fig. 6a-c). The video can be viewed at http://langint.pri.kyoto-u.ac.jp/ai/en/ album/the_drawings_by_chimpanzees.html.

\section{Scarf painted by Ai}

The Ai Project began in 1977 and we will celebrate its 40th anniversary this year, 2017. Prof. Oike proposed that we create a scarf, made out of silk and painted by $\mathrm{Ai}$, as the special anniversary commemorative gift. For this scarf, we used the painting created for Prof. Oike in red and black. Thanks to collaborators at Kyoto University of Arts and Design, we carried out a series of trials and finally succeeded in creating a silk scarf at the end of 2016 (Fig. 7).

Taking the opportunity to visit the UK to give a talk at the Royal Society in February 2017, I visited Jane Goodall, who happened to be at home in Bournemouth. Jane was very happy to receive the silk scarf painted by $\mathrm{Ai}$, a longtime friend of hers (Fig. 8). She has watched the life of Ai and her son Ayumu unfold over the years. This scarf can be viewed as symbolic: a gift from Ai the chimpanzee representing the entire chimpanzee community to thank Jane for her lifelong commitment to the conservation and welfare of chimpanzees.

I hold the post of Editor-in-Chief of Primates, General Director of the Japan Monkey Centre (JMC) and am a Lifetime Professor at Kyoto University Institute for Advanced Study. The JMC is an ideal place for social outreach for researchers of chimpanzees such as those involved in the Ai Project. Jane Goodall recommended to me that I make these unique and striking scarves available to buy for people who are likely to appreciate art by apes. I hope that the scarf design gains widespread attention among people who enjoy the beauty of chimpanzee art. We are also planning to create an art book-a collection of paintings and drawings created by apes-and I hope that the JMC will become a window to allow people to connect with art by apes.

\section{References}

Matsuzawa T (1985) Use of numbers by a chimpanzee. Nature 315:57-59. doi:10.1038/315057a0

Matsuzawa T (1996) From drawing to writing. In: Matsuzawa T (ed) Chimpanzee being, 8th edn. Iwanami-shoten, Tokyo (in Japanese)

Matsuzawa T (2003) The Ai Project: historical and ecological contexts. Anim Cogn 6(4):199-211. doi:10.1007/s10071-0030199-2

Matsuzawa T (2016) SAGA and GAIN for great apes. Primates 57:1-2. doi:10.1007/s10329-015-0504-0

Matsuzawa T (2017) The Nimba Mountains in Guinea. Primates 58:1-3. doi:10.1007/s10329-016-0591-6

Matsuzawa T, Tomonaga M, Tanaka M (2006) Cognitive development in chimpanzees. Springer, Berlin

Matsuzawa T, Humle T, Sugiyama Y (2011) The chimpanzees of Bossou and Nimba. Springer, Berlin

Morris D (1962) Biology of art. Methuen, London

Saito A, Hayashi M, Takeshita H, Matsuzawa T (2014) The origin of representational drawing: a comparison of human children and chimpanzees. Child Dev 85:2232-2246. doi:10.1111/cdev.12319

Tanaka M, Tomonaga M, Matsuzawa T (2003) Finger drawing by infant chimpanzees (Pan troglodytes). Anim Cogn 6:245-251 